\title{
Germanica
}

\section{Modernité Est/Ouest : réceptions et résistances}

\section{Bénédicte Terrisse et Werner Wögerbauer}

\section{OpenEdition}

\section{Journals}

Édition électronique

URL : http://journals.openedition.org/germanica/3336

DOI : 10.4000/germanica.3336

ISSN : 2107-0784

\section{Éditeur}

Université de Lille

\section{Édition imprimée}

Date de publication : 30 décembre 2016

Pagination : 7-17

ISBN : 9782913857384

ISSN : 0984-2632

\section{Référence électronique}

Bénédicte Terrisse et Werner Wögerbauer, « Modernité Est/Ouest : réceptions et résistances », Germanica [En ligne], 59 | 2016, mis en ligne le 03 mars 2017, consulté le 07 janvier 2021. URL : http:// journals.openedition.org/germanica/3336 ; DOI : https://doi.org/10.4000/germanica.3336

(C) Tous droits réservés 


\section{Modernité Est/Ouest : réceptions et résistances}

Les contributions présentées dans ce dossier sont issues d'un atelier doctoral franco-allemand qui s'est tenu à l'université de Nantes le 26 février 2016. Celui-ci se proposait de rouvrir la question du sort de l'héritage de la modernité dans l'Allemagne divisée en revenant sur le postulat communément admis d'une polarisation opposant, à partir des années 1950, une Allemagne de l'Ouest bastion quasi naturel de la modernité à une Allemagne de l'Est s'inscrivant avec obstination du côté de la tradition'1.

Première manifestation du Programme de Formation Recherche 20152017 du CIERA, mené en coopération avec les universités de ParisSorbonne, Iéna et Nantes, et intitulé « Modernité Est-Ouest : Wolfgang Hilbig et (toute) la modernité », l'atelier s'est saisi du premier pan de la question, "Modernité Est-Ouest », à la lumière de laquelle il s'agissait ensuite d'examiner l'œuvre particulière de l'écrivain est-allemand, dont l'ampleur des références à la modernité ne laisse d'étonner. L'objectif était donc dans un premier temps de mettre la problématique historicolittéraire à l'épreuve d'une variété d'œuvres, de courants, d'auteurs et de groupements littéraires de l'Est et de l'Ouest de l'Allemagne et de pratiquer une forme d'histoire littéraire intégrée des deux Allemagnes d'après 1945.

Le présent recueil porte la trace à la fois du projet global et du caractère expérimental de ce premier état des lieux. Si la majorité des contributions ont trait au problème de la modernité après 1945 sans qu'il y soit question de Hilbig, elles concourent cependant aussi à poser les jalons permettant de comprendre la situation historique et littéraire dans laquelle prend place l'œuvre de l'écrivain. Les deux articles faisant le

1. - Wolfgang Emmerich, « Schicksale der Moderne in der DDR », in Sabine Becker/Robert Krause (Hg.), Literarische Moderne. Begriff und Phänomen, Berlin, De Gruyter, 2007, p. 417-434. Cf. p. 427 « [...] verglichen mit der gleichsam geschenkten [Modernität] in der Bundesrepublik » («[...] comparé à la modernité pour ainsi dire offerte en République fédérale »). 
lien entre l'œuvre de Hilbig (1941-2007) et la question de la modernité que nous avons placés à la fin du dossier en fournissent en quelque sorte les exemples.

On relève des usages si variés du concept de « modernité » en littérature qu'il semble impossible d'en donner une définition qui pourrait rendre compte de l'ensemble des acceptions. Le terme fait référence à un découpage chronologique pour désigner des périodes très différentes. Il procède généralement de l'identification d'un moment de rupture : le spectre va du début des temps modernes aux avant-gardes du $\mathrm{XX}^{\mathrm{e}}$ siècle en passant par les transformations esthétiques dans la seconde moitié du XVIII $I^{\mathrm{e}}$ siècle, le romantisme, le post-romantisme et le basculement qui s'est produit autour de $1900^{2}$. Le flottement terminologique se traduit en allemand par une pluralité d'expressions : « Moderne » voisine avec « Modernität »-à côté de « Modernismus », qui est sans doute influencé par l'anglais « modernism »; on peut y ajouter les emplois plus libres de l'adjectif : «moderne Dichtung » ou « moderne Literatur ». Lié à la nouveauté, à l'inédit et la concurrence des modèles esthétiques, le concept peut servir une visée tantôt programmatique, tantôt critique ${ }^{3}$, et permet de proposer une trame à l'historiographie littéraire. Les œuvres s'inscrivent alors dans une sorte de frise chronologique, dont chaque segment possède ses propriétés formelles et thématiques ; le modèle conduit à mesurer leur adéquation aux attentes préétablies, à constater une précocité ou un retard, et permet d'y voir selon les cas un phénomène d'anticipation ou l'expression d'un besoin de rattrapage ${ }^{4}$. Cette " pendule de la modernité » a joué un rôle particulier dans l'art allemand du $\mathrm{XX}^{\mathrm{e}}$ siècle.

En laissant de côté les macropériodisations qui font remonter la modernité à l'apparition des littératures européennes non-latines ou à la Renaissance, il semblerait que la plupart des acceptions se réfèrent à l'apparition ou à la radicalisation de l'idée d'un absolu littéraire, à un moment d'affranchissement et de délimitation de la littérature comme enceinte séparée. Pour la littérature allemande, on a pu souligner le paral-

2. - Victor Žmegač, « Moderne/Modernität », in Dieter Borchmeyer / V. Ž., Moderne Literatur in Grundbegriffen, Tübingen, De Gruyter, 1994, p. 278-285.

3. - Cf. la définition de la modernité comme « Kampfbegriff» (« terme de combat ») du sociologue Wolfgang Knöbl. Il fait des controverses autour de la signification du terme un critère définitionnel de la modernité, celle-ci étant caractérisée moins par son contenu que par son utilisation polémique, normative et distinctive, ainsi que par son caractère " asymétrique », supposant que l'on crée dans le même temps un adversaire que l'on caractérise comme non moderne. Knöbl rappelle en outre que les théories sociologiques ou historiques de la modernité ont vu le jour pour la plupart pendant la Guerre froide. Elles porteraient la marque de ce contexte. Wolfgang Knöbl, « Beobachtungen zum Begriff der Moderne », IASL 37 (2012) 1, p. 63-77.

4. - Sur le caractère problématique du « récit de rattrapage » encore communément utilisé pour décrire le rapport de la RDA à la modernité, cf. Stephan Pabst, Post-OstModerne. Poetik nach der DDR, Göttingen, Wallstein, 2016, p. 406-408. 
lélisme entre la construction de l'État nation et la conception d'une littérature nationale censée la préparer et l'accompagner, voire la suppléer ${ }^{5}$. La réalisation de l'unité nationale marque la fin de ce paradigme, elle ouvre en même temps la perspective d'une histoire littéraire qui ne serait plus l'émanation d'une histoire sociale et politique. Dans cette hypothèse, l'autonomie du champ littéraire conférait aux œuvres un caractère autotélique et autoréférentiel, elle pouvait favoriser l'exploration des pratiques artistiques, leur mise en question méthodique et l'expérimentation de techniques nouvelles dans un rapport à la fois d'émulation et de rivalité entre l'art et le développement simultané des sciences expérimentales.

La modernité artistique, telle qu'elle s'affirme autour de 1900, a été d'abord un phénomène européen et supranational, avant qu'elle ne soit prise dans la tourmente des événements tragiques du $\mathrm{XX}^{\mathrm{e}}$ siècle. Dans le domaine allemand, on suppose généralement qu'il y a une opposition de principe entre l'art moderne et la politique culturelle du nazisme, un antagonisme symbolisé par l'exposition sur l'« art dégénéré » de 1937. La vision est très réductrice - il suffit pour cela de rappeler le débat sur l'expressionnisme qui s'est développé parmi les écrivains et intellectuels en exil, et plus largement, les nombreuses zones de contact entre le régime nazi et les avant-gardes ${ }^{6}$. Mais elle fait partie des représentations qui aident à comprendre les usages du concept de modernité après 1945 en Allemagne. Sur ce point, les chemins des deux Allemagnes se séparent clairement. Il y a eu, à l'Ouest, ce besoin de rattrapage, encouragé par la politique culturelle des alliés, où l'art moderne semblait avoir partie liée avec la démocratie 7 . Il s'agissait de renouer les fils après la coupure provoquée par douze années de nazisme, de s'ouvrir aux tendances venues de l'étranger, de redécouvrir la modernité et de retrouver les réalités d'une vie artistique et littéraire transnationale. À l'Est, après une phase de tolérance, voire d'encouragement de la modernité artistique en zone soviétique, les débuts de la Guerre froide vers 1947 ont marqué l'imposition du réalisme socialiste de l'idéologue stalinien Andreï Jdanov 8 . D'une certaine manière, la construction du socialisme jouait dans le champ

5. - Hans-Robert Jauss, " Literaturgeschichte als Provokation der Literaturwissenschaft » (1967), in H.-R. J., Literaturgeschichte als Provokation, Frankfurt am Main, Suhrkamp, 1970, p. 144-207, voir p. 148-151.

6. - Hans-Jürgen Schmitt (Hrsg.), Die Expressionismusdebatte. Materialien zu einer marxistischen Realismuskonzeption, Frankfurt am Main, Suhrkamp, 1973 ; Peter Ulrich Hein, Die Brücke ins Geisterreich. Künstlerische Avantgarde zwischen Kulturkritik und Faschismus, Reinbek bei Hamburg, Rowohlt, 1992.

7. - Sur les « enjeux politiques des discours en faveur de la modernité, dans un contexte de crispation idéologique entre Américains et Soviétiques », cf. Élise Petit, "Introduction », in E. P. (dir.), La création artistique en Allemagne occupée (19451949). Enjeux esthétiques et politiques, Paris, Delatour France, 2015, p. 9-37, ici p. 9.

8. - Ibid., p. 21. 
littéraire est-allemand le rôle structurant que l'on avait fait tenir au roman national dans les histoires littéraires du XIX ${ }^{\mathrm{e}}$ siècle. On connaît le rejet et la suspicion dont étaient frappés les auteurs du modernisme - Kafka, Proust, pour ne citer que ces deux exemples - dans les régimes du bloc soviétique ${ }^{9}$. On connaît aussi l'ingéniosité de leurs défenseurs, les subterfuges qu'il fallait inventer pour que ces auteurs puissent être édités, lus et étudiés. On trouvera des exemples de cette réception contrariée et embarrassée dans les enquêtes d'archives de la seconde partie du dossier que nous présentons.

À y regarder de près, l'embarras n'était pas moindre du côté de la RFA - ce qui oblige à nuancer le discours du « bastion naturel de la modernité ». En dépit de l'ouverture de principe, une résistance pouvait se manifester, tant du côté du public que de la critique. L'antimodernisme d'un Emil Staiger, qui s'exprima avec fracas dans sa diatribe Literatur und Öffentlichkeit en 1966 au Schauspielhaus de Zurich, n'était pas une exception suisse, elle traduisait une hostilité très présente dans la société ouest-allemande ${ }^{10}$. Cette résistance à la modernité pouvait être due à la défense de valeurs morales conservatrices ; dans d'autres cas, elle s'exprimait dans des prises de position plus théoriques. Le livre de Hugo Friedrich paru en 1956 sur la «structure » de la poésie moderne, rapidement devenu ouvrage de référence, a fixé pour des générations d'étudiants les traits caractéristiques de la modernité, sans que ses lecteurs aient toujours été conscients des intentions polémiques qui étaient au cœur du projet ${ }^{11}$. En rapportant la poésie moderne à la poésie française du dix-neuvième siècle, il la plaçait sous le signe de l'aléatoire et de la virtuosité verbale, qui l'auraient coupée de l'expérience destinale d'un sujet. Les années 1950 ont aussi vu naître des débats sur les métaphores au génitif ( Genitivmetaphern »), pourfendues en raison de la liberté d'agencement où on ne voyait que combinatoire arbitraire ( x-beliebig »). Les préjugés se fortifient dans une sorte de miroir franco-allemand, où la modernité est considérée comme produit d'importation, imitation illégitime de ces «étrangers qui sur les quais de Paris jouent la langue aux dés (à la manière des disciples de Mallarmé et des surréalistes) ${ }^{12}{ }^{2}$.

9. - Cf. l'ouvrage de référence de Günter Erbe, Die verfemte Moderne. Die Auseinandersetzung mit dem « Modernismus » in Kulturpolitik, Literaturwissenschaft und Literatur der DDR, Opladen, Westdeutscher Verlag, 1993.

10. - Walter Höllerer (Hg.), Sprache im technischen Zeitalter. Der Zürcher Literaturstreit. Eine Dokumentation, 1967/22.

11. - Rémy Colombat, " Hugo Friedrich ou les incertitudes de la modernité », Revue d'Allemagne, 16/1984, 4, p. 591-615.

12. - « [...] jenen Fremden, die auf der Kaimauer von Paris mit der Sprache würfeln (wie die Mallarmé-Nachfolger und Surrealisten) », Otto Pöggeler, Spur des Worts. Zur Lyrik Paul Celans, Freiburg im Breisgau, Karl Alber Verlag, 1986, p. 266. 
C'est à cette réactualisation des débats autour de la modernité dans le contexte de la Guerre froide et à l'analyse fine de la manière dont les pratiques corroborent ou invalident les principes politiques et idéologiques établis dans l'Allemagne occupée et figés par la suite en image d'Épinal que s'attachent les différentes contributions du dossier. Chacune tente de jeter une lumière singulière sur un aspect de la question de la modernité, des deux côtés du rideau de fer.

Les articles du dossier s'organisent en trois ensembles. Les deux premières études mettent en regard la réception des deux côtés du rideau de fer d'un même mouvement ou d'un groupement littéraire (le Nouveau Roman et le mouvement dada), tandis que les deux dernières sont consacrées à la lecture de la prose et de la poésie de Hilbig à l'aune de la modernité. Quant aux trois articles placés au milieu du dossier, ils présentent des études de cas révélatrices de la réception de la modernité à l'Est et à l'Ouest, ainsi qu'un retour sur la modernité esthétique autour de 1900 permettant de comprendre ses prolongements dans une œuvre plus tardive.

Si l'approche comparative des deux premières contributions met en évidence les ressemblances plutôt que les différences dans la réception de la modernité à l'Est et à l'Ouest, les textes se distinguent cependant fortement par la perspective adoptée. L'étude de Solange Arber place au centre de l'analyse la réception du Nouveau Roman français en RFA et en RDA. Celle d'Agathe Mareuge adopte le point de vue des acteurs, les dadaïstes vieillissants, en mettant l'accent sur les relations qu'ils entretiennent avec les milieux littéraires ouest- et est-allemands.

La première étude du recueil, celle de Solange Arber (« Le Nouveau Roman, pierre de touche de la modernité littéraire »), est consacrée au transfert littéraire du Nouveau Roman entre la France et les deux Allemagnes. Elle montre comment, de 1950 à 1970, le Nouveau Roman incarne le roman moderne par excellence. Emblème de la modernité littéraire, tant en France qu'en RFA et en RDA, il devient l'étalon à l'aune duquel se mesure le degré de modernité de chaque littérature. Dans ce cas précis, le rapport de l'Allemagne divisée à la modernité passe par des médiations. Il fait entrer en jeu un quatrième terme, la France, qui incarne aux yeux de nombreux intellectuels de l'époque l'avant-poste de la littérature, d'où est issu le courant novateur, mais également la Suisse et les pays du Bloc de l'Est qui, chacun, joue le rôle de facilitateur dans l'importation du Nouveau Roman en RFA et en RDA. Ce modèle aux actants multiples éclaire singulièrement la question de la réception de la modernité dans l'Allemagne divisée. L'article met également au jour l'ambivalence fondamentale du regard porté après-guerre sur la modernité. S'inscrivant dans ce qu'à la suite d'Antoine Compagnon Solange Arber appelle le « récit orthodoxe de la modernité », formule qui désigne 
une forme de « purification de l'art », de sa « réduction à l'essentiel ${ }^{13}$ », le roman moderne incarné par le Nouveau Roman fait à la fois l'objet de fascination et de rejet. En retraçant les étapes du transfert du Nouveau Roman à la RFA et à la RDA, l'article parvient cependant à montrer que derrière la répartition attendue des rôles, d'une Allemagne de l'Ouest fascinée s'opposant à une Allemagne de l'Est rétive et condamnant toute innovation formelle comme « décadente » et « formaliste », les discours conservateurs (de RFA) et marxistes (de RDA) finissent par se rejoindre dans une méfiance commune à l'égard du Nouveau Roman. En outre, un certain nombre de schèmes mis en œuvre pour appréhender ce roman moderne se retrouvent à l'Est comme à l'Ouest. Par exemple, les stratégies discursives développées par les romanistes et critiques est-allemands pour permettre la réception du Nouveau Roman en RDA malgré la contradiction avec la ligne officielle de la politique culturelle interrogent le rapport entre la notion de modernité esthétique et celle de modernité sociétale : selon eux, la modernité formelle du Nouveau Roman est à mettre en relation avec l'aliénation régnant dans le monde occidental moderne, c'est-à-dire capitaliste, dont il est issu. Néanmoins, le peu d'émules que fait le Nouveau Roman parmi les écrivains de part et d'autre du rideau de fer témoigne d'une résistance à la littérature expérimentale à laquelle on identifie la littérature moderne. Cette résistance, qui s'avère commune à la RFA et à la RDA, met en évidence la tension entre éthique et esthétique propre à la réception de la modernité dans une Allemagne d'après 1945 qui entend rompre avec le passé national-socialiste en pratiquant une littérature de l'engagement perçue comme peu compatible avec les expérimentations formelles du Nouveau Roman. Ainsi, la réception du Nouveau Roman traduit-elle une perception globalement critique de la modernité, qui est emblématique de l'Allemagne divisée. Cette critique de la modernité s'exerce à l'encontre du Nouveau Roman à travers le triple prisme de ses rapports à la réalité, au lecteur et à l'humain. Quoique développés dans des contextes et selon des modalités différentes à l'Ouest et à l'Est, les griefs formulés à l'aide de ces catégories dessinent les contours du problème de la modernité après 1945 (la littérature comme reflet du réel versus autonomie de la littérature, hermétisme versus engagement). Dans le même temps, ils constituent un terrain où se rencontrent et s'affrontent les théories littéraires de l'Est et de l'Ouest (le réalisme socialiste, les théories de la réception de l'École de Constance).

La contribution d'Agathe Mareuge («Quelques aspects des relations entre avant-garde et modernité dans le contexte de l'Allemagne divisée : le cas des dadaiistes vieillissants ») décrit la situation paradoxale des représentants du mouvement dada dans les années 1950 et 1960, dont certains

13. - Antoine Compagnon, Les cinq paradoxes de la modernité, Paris, Le Seuil, 1990, p. 56. 
(parmi lesquels Hans Arp, Raoul Hausmann et Richard Huelsenbeck) se font alors historiens de leur propre mouvement et s'efforcent de l'inscrire dans une continuité qui tranche avec la posture de marginalité et de radicalité adoptée lors de son apparition. Dans un contexte politique et culturel entièrement transformé, le regard rétrospectif de l'avant-garde dada sur elle-même cherche les fils qui pourraient la relier à l'histoire de la modernité et tente de repérer d'éventuels prolongements dans la production artistique contemporaine. En réinterprétant leur passé, les dadaïstes se réapproprient une tradition littéraire spécifique par une forme de recherche généalogique, dans une démarche analogue à celle qu'avait choisie dès 1939 André Breton pour le mouvement surréaliste avec son Anthologie de l'humour noir. Pour l'Allemagne de l'Ouest, Agathe Mareuge met en évidence un intérêt grandissant pour le mouvement dada dès la fin des années 1950, et décrit les conditions dans lesquelles les ouvrages des dadaïstes « survivants » sont alors accueillis dans le monde éditorial et littéraire, en particulier dans des revues comme Akzente et Sprache im technischen Zeitalter. La réception de dada a été plus tardive en RDA, avec des effets très différents de ceux que l'on pouvait observer en RFA, puisqu'elle a pu encourager à l'Est à partir des années 1980 la formation d'une vie littéraire en marge des institutions, par exemple dans les milieux du Prenzlauer Berg, qui s'inspiraient du potentiel subversif des avant-gardes historiques pour mieux exprimer leur opposition à la culture officielle.

À première vue, il pourrait paraitre surprenant de revenir dans un dossier consacré à la situation de la modernité dans l'Allemagne divisée, sur le débat qu'a suscité dans les années 1930 l'œuvre d'un écrivain du XIX ${ }^{\mathrm{e}}$ siècle parmi les écrivains et intellectuels allemands de l'exil. Il s'agit, d'une part, du conflit d'interprétation qui a opposé le philosophe Georg Lukács au germaniste Karl Viëtor (dont la lecture conservatrice a été accusée, sans doute à tort, de tendances «fascisantes ») et d'autre part de l'imposante monographie rédigée pendant ces mêmes années (elle ne paraîtra qu'après-guerre) de Hans Mayer, Georg Büchner und seine Zeit. La question posée était celle d'une lecture marxiste de Büchner. La contribution de Laetitia Devos (« Lectures marxistes de La Mort de Danton de Büchner : interprétations de Georg Lukács et Hans Mayer autour de 1935 et leur réception en RDA ») reconstitue les discussions et situe les positions dans le contexte de leur époque. Contre l'interprétation fataliste défendue par Viëtor et d'autres, Lukács et Mayer soulignaient les engagements politiques et les fondements matérialistes du théâtre de Büchner, tout en montrant la complexité de la réflexion sur le processus révolutionnaire qu'il exprime en particulier dans le drame historique $L a$ Mort de Danton. Or, c'est une œuvre qui est aussi fondatrice de la modernité littéraire. Les ruptures esthétiques et formelles qu'elle opère sont si fortes qu'elles peuvent à elles seules expliquer le retard de réception qui 
tend à faire de Büchner, pourtant si intimement lié à l'histoire sociale et politique de son temps, un auteur du $\mathrm{XX}^{\mathrm{e}}$ siècle. Considéré à la fois comme un précurseur de Marx et comme un inventeur des formes dramatiques les plus actuelles, Büchner révélera ensuite les relations tourmentées et contradictoires de la politique culturelle est-allemande à la littérature moderne. Laetitia Devos retrace le développement ultérieur et la réception du débat, les simplifications et les réductions qu'il a subies, et éclaire par ce moyen le climat intellectuel régnant en RDA, en particulier après la disgrâce de Lukács à la suite du soulèvement hongrois en 1956 et le départ pour l'Allemagne de l'Ouest de Hans Mayer en 1959. Les analyses dialectiques proposées par Lukács et Mayer avaient fini par provoquer un malaise qui s'est manifesté dès les années 1960 dans des mises en scène qui modifiaient le texte de Büchner pour mieux le faire correspondre à une vision linéaire et dogmatique de l'histoire révolutionnaire. D'autre part, elles ont pu encourager plus tard l'émergence, au sein de la RDA, de positions contestataires et permettre à une nouvelle génération d'écrivains de se saisir de la figure de Büchner pour affirmer une opposition esthétique autant que politique.

La présentation de l'œuvre de Karl Wolfskehl (1869-1948) par Sonia Schott («Karl Wolfskehl et la modernité poétique ») fournit l'occasion de s'interroger sur les modalités de son inscription dans la modernité poétique. Elle propose un éclairage des transferts culturels qui caractérisent l'intégration du symbolisme français dans le champ littéraire allemand et du rôle tenu par le cercle de Stefan George, qui, bien au-delà de la mort du maître en 1933, a exercé une influence parfois décisive et souvent souterraine sur la culture politique et artistique de la jeune République fédérale ${ }^{14}$. Les analyses de poèmes rendent compte de l'impact que la révolution symboliste a pu avoir en Allemagne à travers ces médiations. Par ailleurs, en s'appuyant sur les critères de modernité dégagés par Hugo Friedrich en 1956, l'étude illustre le paradoxe d'un livre qui, bien que fondamentalement hostile à la poésie moderne, s'est imposé, par un succès de librairie de longue durée, comme une grille de lecture privilégiée de cette poésie en Allemagne.

La contribution de Clément Fradin («Paul Celan ou les affres de la modernité ») montre comment le débat sur la modernité poétique a pu orienter la réception de l'œuvre de Celan en Allemagne de l'Ouest. Il met en évidence les « schèmes d'entendement » qui ont conduit la critique contemporaine à assimiler Celan à une forme de «poésie pure » que l'on rapportait, là encore, au symbolisme français ; dans le même temps, l'article revient sur la manière dont Celan a répondu à ces assignations, aux approximations et aux faux procès qui en découlaient. On reprochait à la

14. - Ulrich Raulff, Kreis ohne Meister. Stefan Georges Nachleben, München, C. H. Beck, 2009. 
poésie de Celan son caractère arbitraire et son manque d'authenticité, ce qui prouve d'ailleurs que l'ouvrage de Hugo Friedrich n'était finalement qu'une manifestation parmi d'autres d'un antimodernisme très répandu. Méconnaissant ou occultant la référence historique qui était le fondement même de la poésie de Celan, on considérait qu'elle avait coupé tout lien avec la réalité, qu'elle « agissait dans le vide ». Clément Fradin analyse les comptes-rendus des recueils parus dans les années 1950 et 1960, et les confronte aux commentaires et répliques formulés par Celan dans sa correspondance et dans un certain nombre de notes publiées de manière posthume. Il esquisse également l'hypothèse d'une référentialité très particulière, qui se serait fortement développée dans les derniers recueils, et qui pourrait bien être le signe d'une modernité qui s'affirme contre les conceptions de la modernité auxquelles ont tentait de réduire son œuvre.

Dans l'avant-dernière contribution de ce recueil, Florian Gödel (« Eine Kiste, deren Inhalt nicht mehr zugänglich ist - Wolfgang Hilbigs Das Provisorium und sein Umgang mit der Shoah ») éclaire un nouvel aspect de la question de la modernité dans l'Allemagne divisée : son rapport aux violences historiques du $\mathrm{XX}^{\mathrm{e}}$ siècle, en particulier la Shoah, à travers l'analyse du dernier roman de l'écrivain d'origine est-allemande, Wolfgang Hilbig, immigré en RFA en 1985. La comparaison entre les deux systèmes de société qui constituent l'Allemagne divisée est inscrite dans l'intrigue même du roman Das Provisorium (Provisoire, 2001). En effet, celui-ci raconte les allers-retours incessants du personnage principal C., écrivain originaire de RDA et alter ego de l'auteur, entre l'Est et l'Ouest de l'Allemagne, entre 1985 et 1989. La découverte de la valeur dominante et normative que revêtent sur le marché du livre ouest-allemand les ouvrages sur la Shoah et le Goulag produit l'effet d'un choc sur le personnage qui est un adepte de la modernité littéraire. Florian Gödel parvient à montrer comment, dans le roman, discours sur la Shoah et discours sur la modernité sont mis en parallèle. Le discours sur la Shoah apparaît tour à tour comme ce qui rend caduc ou justifie celui sur la modernité. Le problème de l'écriture après Auschwitz que pose Adorno apparaît en effet comme le filtre implicite à travers lequel le personnage de $\mathrm{C}$. interroge à nouveaux frais sa légitimité d'écrivain dans le contexte de la RFA. Un parallélisme entre les livres d'écrivains de la modernité internationale, tels Rimbaud, les surréalistes, Arthur Cravan, Kavafis, T.S. Eliot, Breton ou Beckett, et les deux caisses non ouvertes d'ouvrages sur la Shoah et le Goulag, traverse en filigrane le roman. Les effets de la prise de conscience de l'importance de la Shoah sur l'écriture sont décrits dans les mêmes termes que ceux de la crise du langage et de la crise du sujet de la modernité autour de 1900. Désormais, un soupçon pèse sur la capacité de la littérature à dire vrai et à représenter l'irreprésentable des camps de la mort. On remet en cause la possibilité de distinguer la fiction du mensonge. Ce soupçon légitimerait la recherche de formes inédites, 
non mimétiques du réel, emblématiques, en somme, de la modernité. Cette modernité littéraire apparaîtrait en ce sens comme une conséquence des violences historiques du $\mathrm{XX}^{\mathrm{e}}$ siècle, tandis que, dans une perspective là encore toute adornienne, la modernité technique et historique, à travers l'idéologie du progrès et son emblème qu'est le train, semble avoir produit les camps de la mort. La double perspective est- et ouest-allemande éclaire là aussi la question des crimes de l'histoire en ce que le Goulag est traité dans le roman comme une sorte de variante soviétique de la Shoah. L'histoire intégrée des camps que le roman pratique sauvagement lui fait courir le risque du relativisme. Cependant, à travers la question du lien entre écriture moderne et Shoah, à travers les parallèles douteux entre la société de consommation ouest-allemande et Auschwitz, le roman pointe implicitement vers la question du possible traumatisme d'une vie passée sous le régime de mensonge et de manipulation idéologique qu'est la RDA, ainsi que de son impact sur l'écriture. Il soulève la question de l'inscription du régime du SED dans l'histoire des violences étatiques du $\mathrm{XX}^{\mathrm{e}}$ siècle et étudie les ravages de l'idéologie sur un individu.

C'est également l'individu qui est au cœur du deuxième article consacré à l'œuvre de Wolfgang Hilbig. En s'attachant à l'autre bout de l'œuvre de l'écrivain, son œuvre de jeunesse, et à l'autre genre qu'il pratique, la poésie, Jonathan Scholbach (« 'und müde bin ich mir selbst / entflohn'. Negative Identitätskonstruktionen in Wolfgang Hilbigs Gedichtband abwesenheit ») met en évidence, dans le premier recueil de poésie de Hilbig, abwesenheit (1979), un procédé fondamental de l'écriture qui s'inscrit à la suite de l'aliénation du moi et du monde, typique de la modernité esthétique autour de 1900. L'analyse immanente de poèmes permet de mettre au jour plusieurs procédés que Scholbach subsume sous l'appellation de constructions négatives de l'identité. Mettre au jour ces procédés, tels le paradoxe, la négation et la scission, qui concourent à l'obscurité, à la contradiction ou à l'ambiguïté dans les poèmes, permet d'en proposer une interprétation inédite. En effet, la contradiction interne des textes est en partie levée lorsque l'on identifie dans les poèmes la présence de deux instances du moi, la voix qui parle (res loquens) et le corps et le monde physique (res extensa). Ce dédoublement du moi lyrique est le signe que la parole poétique s'émancipe du monde. Cependant l'accent, chez Hilbig, n'est pas mis sur la proclamation emphatique de l'autonomie, critère de la modernité, mais sur le processus d'aliénation qui sépare le moi de son environnement. La lecture immanente fait abstraction du contexte est-allemand et de la biographie de l'ouvrier-écrivain dans une sorte de fiction théorique qui permet de tester d'autres modèles interprétatifs en évitant les ornières de la critique hilbigienne. Néanmoins, le schème de l'aliénation proposé par Jonathan Scholbach n'est pas incompatible avec le vécu de l'écrivain en RDA. Dès lors, la poésie de Hilbig se 
lirait comme la transposition et l'adaptation au contexte de l'Allemagne divisée de procédés et modèles venus de la modernité esthétique.

Nous remercions le CIERA, mais aussi les partenaires du programme, en particulier Sylvie Arlaud et Bernard Banoun (Paris-Sorbonne) et Stephan Pabst (Friedrich-Schiller-Universität Jena), ainsi que la rédaction de la revue Germanica qui nous a proposé d'accueillir les résultats de l'atelier et a relu avec attention et patience les articles.

Bénédicte TERRISSE et Werner WöGERBAUER Université de Nantes 